\title{
Study of the cosmic muon rate nearby the Advanced Virgo detector at the end of the 03 run
}

\author{
Francesco Di Renzo, ${ }^{a, b, *}$ Nicolas Arnaud, ${ }^{c, d}$ Theodoros Avgitas, ${ }^{d}$ Rosario De Rosa, ${ }^{e}$ \\ Irene Fiori, ${ }^{d}$ Carlo Giunchi, ${ }^{f}$ Kamiel Janssens, ${ }^{g}$ Stavros Katsanevas, ${ }^{d}$ Alessandro \\ Longo, ${ }^{h}$ Jacques Marteau, ${ }^{i}$ Marco Olivieri, ${ }^{j}$ Federico Paoletti, ${ }^{b, d}$ Paolo Ruggi $^{d}$ and \\ Maria Concetta Tringali ${ }^{d}$ \\ ${ }^{a}$ Università di Pisa, I-56127 Pisa, Italy \\ ${ }^{b}$ Istituto Nazionale di Fisica Nucleare, Sezione di Pisa, I-56127 Pisa, Italy \\ ${ }^{c}$ Universite Paris-Saclay, CNRS/IN2P3, IJCLab, 91405 Orsay, France \\ ${ }^{d}$ European Gravitational Observatory (EGO), I-56021 Cascina, Pisa, Italy \\ e Università di Napoli “Federico II”, I-80126 Napoli, Italy \\ ${ }^{f}$ Istituto Nazionale di Geofisica e Vulcanologia, Sezione di Pisa, I-56125 Pisa, Italy \\ ${ }^{g}$ Universiteit Antwerpen, Prinsstraat 13, 2000 Antwerpen, Belgium \\ ${ }^{h}$ Istituto Nazionale di Fisica Nucleare, Sezione di Roma Tre, I-00146 Roma, Italy \\ ${ }^{i}$ Institut de Physique Nucléaire de Lyon, Lyon, France \\ ${ }^{j}$ Istituto Nazionale di Geofisica e Vulcanologia, Sezione di Bologna, I-40128 Bologna, Italy \\ E-mail: francesco.direnzo@df.unipi.it
}

Cosmic-ray particles have long been studied as a potential source of noise for interferometric gravitational-wave detectors. These particles, mostly muons at sea level, can interact with the detector mirrors inducing thermal effects, which, at the detector output, could be observed as transient excesses of noise, namely glitches. For the Advanced Virgo detector, the rate of these particles is monitored by a muon telescope located in the vicinity of the detector central building. We present here the correlation study of the rate of muons with the rate of glitches during a couple of weeks at the end of the third joint LIGO-Virgo observing, O3. We also present the correlation of the previous quantities with other environmental effects, showing how the latter dominate the glitch rate and can explain a significant part of its variations.

\footnotetext{
*** The European Physical Society Conference on High Energy Physics (EPS-HEP2021), ***

*** 26-30 July $2021 * * *$

*** Online conference, jointly organized by Universität Hamburg and the research center DESY ***
}

\footnotetext{
${ }^{*}$ Speaker
} 


\section{Introduction}

Ground-based gravitational-wave (GW) detectors, like Advanced Virgo [1], are constantly passed through by cosmic rays, of which muons constitute the most abundant charged particles at sea level [2]. These can interact with the detector mirrors producing the excitation of their vibrational modes [3]. Depending on the rate of particles and the decay time of these modes, the resulting effect can appear as a transient excess of power, called glitch [4], or as an additional background noise. This effect has long been investigated in the literature [5] to understand its relevance for GW searches.

We present here the first experimental results about the impact of these particles on detector noise based on the data jointly collected for about 17 days by Advanced Virgo and a muon telescope installed close to the Virgo beam splitter mirror [6].

\section{Muon rate in the correspondence of candidate events}

Our first test is to verify whether the muon rate in the correspondence of candidate $G W$ events is consistent with its reference values of the period; if an unusually high rate is measured, we may expect more transient noise affecting these events.

Four of such events have been published on the Gravitational-Wave Candidate Event Database (GraceDB), ${ }^{1}$ plus more are expected to be identified from offline analyses. For each of them, we estimate the empirical cumulative distribution function (e.c.d.f.) of the counts per second of muons in one hour around the time of the event, excluding the second of the event. The percentile corresponding to counts in the latter is interpolated form the e.c.d.f., from which we obtain its $p$-value. The null hypothesis that the rate is consistent with the expectation for that period has to be rejected if the $p$-value exceeds a test significance decided in advance, say $1 \%$ or $5 \%$. None of the examined events led to a rejection of this hypothesis; we have no evidence that the rates were larger than ordinary. We also performed a joint hypothesis test with the Fisher's method [7], combining the $p$-values of all of the candidate events. The resulting $p$-value is about $36.1 \%$, which supports what we have obtained examining each event independently.

\section{Correlation between muon and glitch rates}

The second test that we present is a correlation analysis of the rate of muons and that of glitches in Virgo GW data. Firstly, we have verified that the variability of the former is consistent with that of a Poisson distributed random process over time scales shorter than $\sim 6$ hours. Estimating the mean of this quantity on shorter periods provides a statistic that highlights the physical changes, allowing to ignore its inherent variability. The average minute rates, estimated over intervals of 30 minutes, are represented by the red and blue lines, for muons and glitches respectively, in the left-hand side part of Fig. 1. The cross-correlation between the two time series is reported in the 2D-histogram on the right-hand side of Fig. 1. A Pearson correlation coefficient $r$ of 0.76 highlights a quite large value of this correlation, which however doesn't tell anything about a potential causation mechanism for which the glitches are consequence of the muons.

1https://gracedb.ligo.org/ 

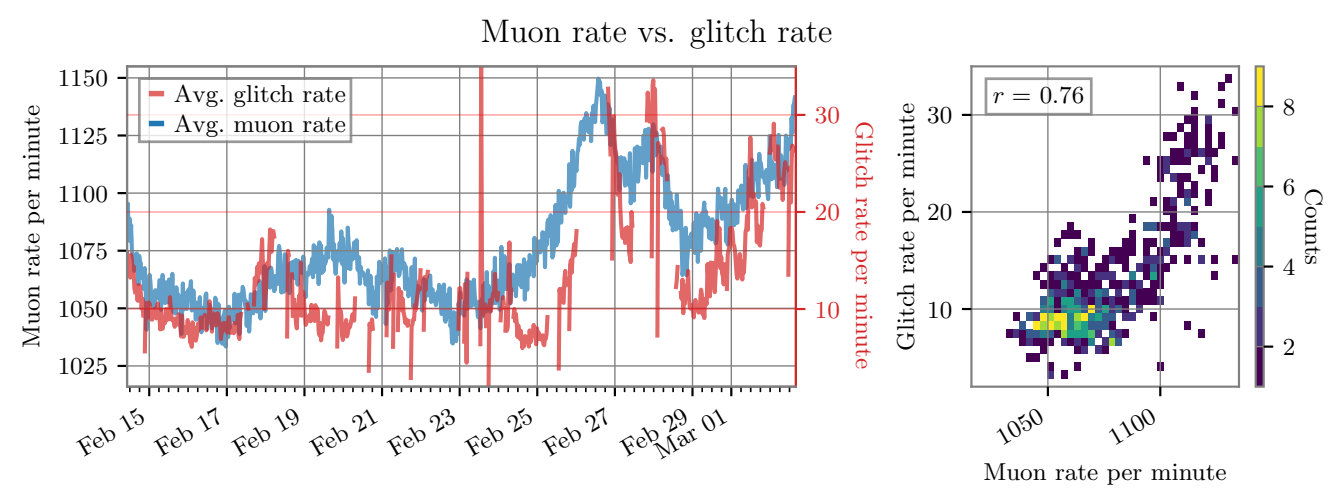

Figure 1: Correlation between muon and glitch rates. In the left-hand side plot, the time series of the average rate per minute of muons and glitches are represented by the solid blue and red lines respectively. On the right-hand side, the 2D-histogram shows their correlation, and in the box on the top left corner is reported the corresponding Pearson correlation coefficient $r$.

\section{Impact of atmospheric conditions on muon and glitch rates}

To correctly interpret the previous result, we have to understand what produces the variations of the muon rate. Indeed, it is well known that this is inherently highly dependent on the weather conditions, in particular pressure and temperature [8]. We verified this relation by means of a multi-variate linear regression of the averaged muon rate per minute and the weather monitors from the meteo station located nearby the Virgo Central Building. The optimal solution, obtained via the Ordinary Least Square (OLS) method produced an impressing $R^{2}$ determination coefficient of $92.2 \%$, meaning that such percentage of the muon rate variability can be modeled with the variations of the weather conditions. The great agreement of the model (red line) with the data (blue dots) is visible in Fig. 2, where the red band is the prediction confidence interval with significance $\alpha=5 \%$. In particular, we verified that the muon rate is (anti-)correlated with the atmospheric pressure and temperature. The glitch rate is likewise affected by weather conditions. In particular, differences in atmospheric temperature let the warm air move up, with a consequent reduction of pressure at ground level, and a wind from the higher to the lower pressure area. This wind is also a known cause of microseismic noise and glitches in GW data, hence the positive correlation between glitch and muon rates [4].

In an attempt to disentangle this effect from both rates, we proceed to repeat the OLS regression also for the glitches. Then, we evaluate the correlation of the residuals of both regressions, where the weather contributions is assumed to be no more present. This produces a Pearson's $r \simeq 15 \%$. In this case, the lack of correlation in the changes of these two quantities reveals no evidence of causation between them as well.

\section{Conclusions and outlook}

From this joint analysis of muon rates and Virgo data, we have found no evidence that muon interactions can affect the detector noise. The manifest correlation of the two rates provides an interesting example where mutual causes play an important role in the investigation of causation 


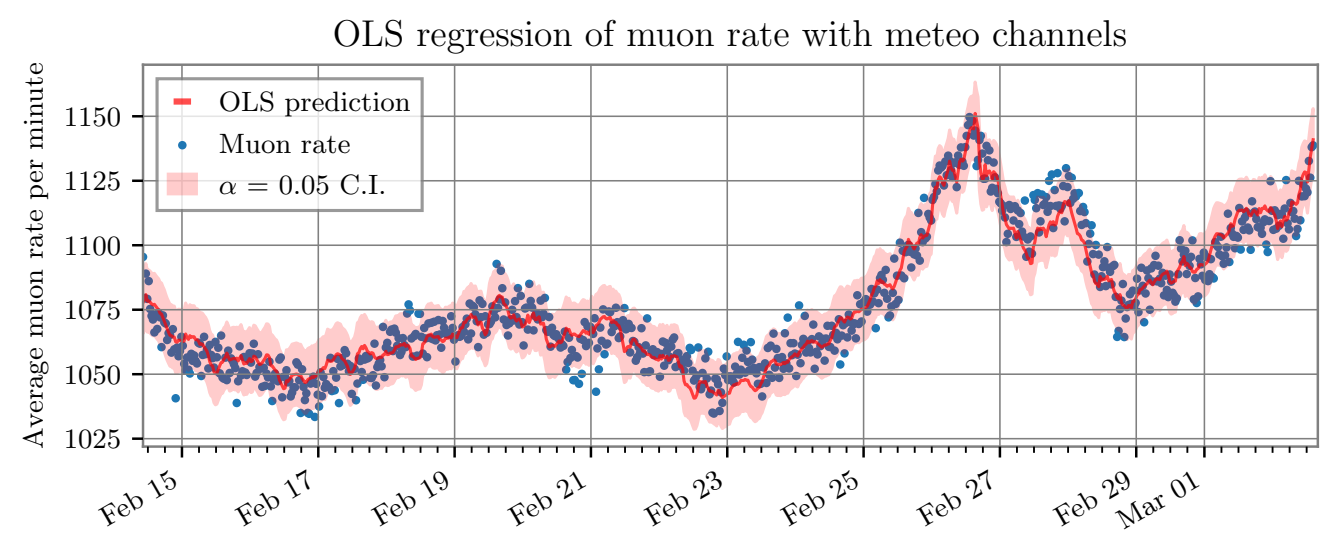

Figure 2: Regression of the average muon rate per minute with the weather sensors from the Virgo meteo station. The blue dots represent the muon rate, while the solid red line is its best OLS prediction making use of the environmental channels. The red band represent the confidence interval, C.I., for this model with a significance $\alpha=5 \%$.

relations. Nonetheless, this is just a first, preliminary result from experimental data. More insight will be possible making use of multiple muon telescopes, able to provide a better coverage of the detector area and muon rate estimates, and also account for the effects of cosmic ray showers [2].

\section{References}

[1] VIRgo collaboration, The Advanced Virgo detector, J. Phys. Conf. Ser. 610 (2015) 012014.

[2] D. Maurin, F. Melot and R. Taillet, A database of charged cosmic rays, Astronomy \& Astrophysics $\mathbf{5 6 9}$ (2014) A32.

[3] B.L. Beron and R. Hofstadter, Generation of mechanical vibrations by penetrating particles, Phys. Rev. Lett. 23 (1969) 184.

[4] VIRGO collaboration, The characterization of Virgo data and its impact on gravitational-wave searches, Class. Quant. Grav. 29 (2012) 155002.

[5] A. Giazotto, Estimate of the cosmic ray background in an interferometric antenna for gravitational wave detection, Physics Letters A 128 (1988) 241.

[6] N. Lesparre et al., Design and operation of a field telescope for cosmic ray geophysical tomography, Geoscientific Instrumentation, Methods and Data Systems 1 (2012) 33.

[7] E. Rödel, Fisher, R. A.: Statistical methods for research workers, 14. aufl., Oliver \& Boyd, Edinburgh, London 1970. xiii, 362 s., 12 abb., 74 tab., 40 s, Biometrische Zeitschrift 13 (1971) 429.

[8] R.R.S. De Mendonça et al., Analysis of atmospheric pressure and temperature effects on cosmic ray measurements, Journal of Geophysical Research: Space Physics 118 (2013) 1403. 